\title{
EFFECT OF PHYSICOCHEMICAL PROPERTIES OF EMULSIONS FORMED BY SELF-EMULSIFYING DRUG DELIVERY SYSTEMS (SEDDS) ON THE SOLUBILIZATION STATE OF DRUG: IN VITRO STUDY
}

\author{
NASER M. Y. HASAN ${ }^{*}$ \\ *School of Pharmacy, Applied Science Private University, Jordan \\ Email: n_hassan@asu.edu.jo \\ Received: 22 Jul 2018, Revised and Accepted: 19 Nov 2018
}

\begin{abstract}
Objective: Parameters in the oil pre-concentrate which can affect the solvent capacity of the resultant dispersion such as, oil-cosurfactant ratio, type of surfactant used in the system, the inclusion of water soluble co-solvents and the solubilization capacity of native surfactants such as, bile salts and lecithin were studied in an attempt to circumvent crystallization of drug during its passage in the gut.

Methods: Different types of self-emulsifying systems representing type II, IIIA and IIIB, were used to probe the influence of the various physicochemical properties of the resultant dispersions on the fate of dissolved model lipophilic drug. This was achieved by studying emulsification behavior of lipid systems in fed and fasted biological fluids, analyzing solubilization/drug crystallization kinetics and oil droplet diameter measurement.

Results: Self-micro-emulsifying lipid systems lost solvent capacity on dispersion and were not able to keep the drug in solution at equilibrium. Miglyol 812/Imwitor ratio in the pre-concentrate mixture appeared to influence the kinetics of drug crystallization. Pre-microemulsion systems containing Tagat TO dispersions were found to hold more drugs in solution at equilibrium than in the case of systems containing Cremophor RH40. The inclusion of as little as 10-20\% PEG in the lipid mixture accelerated drug precipitation. Bile salt-lecithin mixed micelles appears to some extent enhance the solubilization capacity of these systems after dispersion
\end{abstract}

Conclusion: Solvency of emulsions formed by self-emulsifying drug delivery in various emulsification media is a crucial parameter influencing the fate of dissolved drug after the dispersion of the formulations.

Keywords: SEDDS, SMEDDS, Lipid formulations, Medium chain mono-and glycerides, Poorly water-soluble compounds

(C) 2019 The Authors. Published by Innovare Academic Sciences Pvt Ltd. This is an open-access article under the CC BY license (http://creativecommons.org/licenses/by/4.0/) DOI: http://dx.doi.org/10.22159/ijap.2019v11i1.28664

\section{INTRODUCTION}

Lipid-based drug delivery systems (LBDDS) including selfemulsifying drug delivery systems (SEDDS) or self-microemulsifying drug delivery system (SMEDDS) are isotropic mixtures of oils and non-ionic surfactants which upon gentle agitation in water produce $(\mathrm{o} / \mathrm{w})$ dispersions of droplets $<5 \mu \mathrm{m}[1]$ or between 5 and $140 \mathrm{~nm}$ [2], respectively. Due to high hydrophilic content of SMEDDS which include materials such as; polar oils including mixed mono and/or di-medium chain glycerides, high HLB nonionic surfactants (>12), and hydrophilic co-solvents, these oil vehicles are considered more hydrophilic than SEDDS. Oil-based systems were classified into type I, II, IIIA, IIIB and IV, based on various physicochemical factors such as; the hydrophilicity of the oil mixture, the particle size of the resultant dispersion and the formulation digestibility [3-4]. Type IV formulations do not contain natural lipids and represent the most hydrophilic formulations. Hydrophilicity of the lipid mixture increases by moving from type 1 lipid class system to type 4 . The reformulation of cyclosporine $A$ as Neoral ${ }^{\circledR}$ is an archetypal example of a Type III system [5]. The HIV protease inhibitor amprenavir (Agenerase ${ }^{\circledR}$ ) which contains TPGS as a surfactant and PEG 400 and propylene glycol as co-solvents [6] is an example of Type IV formulations which do not contain natural lipids and thus represent the most hydrophilic formulations. Amprenavir (Agenerase ${ }^{\circledR}$ ) was succeeded though by a pro-drug, fosamprenavir [7]. These lipid formulations are thoroughly characterized and studied, and hence represent one of quintessential approach for the bioavailability enhancement poorlywater-soluble compounds; especially class II drugs. The past nearly 4 decades from 1975 through 2013 have shown rampant growth in utilizing solubilization techniques, accounting on average for around $6 \%$ of all new molecular entities (NMEs) approved [8]. Furthermore, the same study has shown that LBDDS are the most widely used solubilization platform. It is estimated that LBDDS oral drug products account for $2-4 \%$ of all commercially available drug products according to a study by Strickley in 2007 [9]. According to various drug databases and reviews, there are more than 36 different oral LBDDS on the market of 27 unique drug molecules that were FDA approved by FDA [10-12]. Some of the most recent LBDDS products include; Isotretinoin (Absorica ${ }^{\circledR}$, by Cipher pharmaceuticals a reformulation of isotretinoin (Accutane); New drug application year NDA; 2012) [13], Enzalutamide (Xtandi®, NDA; 2012) [14], Nintedanib (Ofev®, NDA; 2014) [15] and Calcifediol (Rayaldee ${ }^{\mathrm{TM}}$, NDA; 2016) [16].

In the design of successful lipid formulations with maximize the bioavailability; key elements in the lipid composite have to be optimized [17-18]. Nonetheless, crystallization of the drug in the lumen of the gut depends on the hydrophilicity of oil system; $\log P$ of the drug and the solubilization capacity of native surfactants (bile salt-lecithin mixed micelles) to maintain the drug in solution during digestion. The medium into which the drug must dissolve has a great influence on its solubility hence; suitable media which satisfactorily simulate the physiological conditions are needed. In the GI tract, the solubility of the drug is a function of aqueous solubility, crystallinity, drug lipophilicity, $\mathrm{p} K_{\mathrm{a}}$ in relation to the $\mathrm{pH}$ profile of GI tract, solubilization by native surfactants (such as bile salts and lecithin) and ingested food components. Four suitable media have been suggested to simulate the composition of proximal GI tract [19]: SGF plus surfactant for fasted state stomach; long-life milk (3.5\% fat) for fed state stomach; FaSSIF and FeSSIF for fasted and fed state small intestine. Physicochemical properties of emulsions formed by various types of lipid systems which can influence drug solubilization were studied here in an attempt to probe the fate of dissolved drug after dispersion.

\section{MATERIALS AND METHODS}

\section{Materials}

Miglyol 812 (medium chain triglyceride) and Imwitor $988\left(\mathrm{C}_{8} / \mathrm{C}_{10}\right.$ mono/diglycerides) were supplied by Condea Chemie $\mathrm{GmbH}$. Tagat TO (PEG-(25)-glyceryl trioleate) was supplied by Goldschmidt AG, 
Germany. Crillet 4 (Polyoxyethylene-(20)-sorbitan monooleate), also known as Tween 80 or polysorbate 80 , was supplied by Croda Chemicals. Cremophor RH 40 (polyoxyethylene-(40)-hydrogenated caster oil) and Cremophor EL (polyoxyethylene-(35)-caster oil) were obtained from BASF Corporation. Hydrophilic co-solvents used in lipid formulations; Propylene Glycol (1,2,-Propanediol) and Polyethylene Glycol 400 were purchased from Sigma, UK; Ethanol 96\% w/w GPR, BDH Chemicals; Transcutol $\mathrm{P}^{\circledR}$ (diethylene glycol monoethyl ether or Ethoxydiglycerol) was obtained from Gattefossé Corporation and Glycofurol ( $\alpha$-Tetarahydro furanyl-w-hydroxy-poly (oxyethylene) was supplied by Roche, Switzerland. Methanol $96 \%$ v/v GPR, BDH Chemicals was used as a solvent for the analytical assays. Materials used to prepare the simulated fasted and fed state intestinal conditions (FaSSIF and FeSSIF); Na Taurcholate, Lecithin, and KCL were obtained from Sigma and $\mathrm{KH}_{2} \mathrm{PO}_{4}$ from $\mathrm{BDH}$
Chemicals. Dimethyl aminoazobenzene also was known as Dimethy Yellow was used in this study as a model drug of 'poorly soluble' weak base ( $\log \mathrm{P}$ of 4.52, pKa of 3.226 and $\mathrm{S}_{0}=$ $1.33 \mu \mathrm{g} / \mathrm{ml}$ ) and was purchased from Sigma, UK. All water used was milli Q water

\section{Methods}

\section{Preparation of the lipid mixtures}

Mixtures of oil, co-surfactant, and surfactants representing Type I, II, IIIA and IIIB were produced by accurately weighing ingredients into screw-capped glass vials with tight closures, see table 1 . Mixtures were placed in a water bath at $50{ }^{\circ} \mathrm{C}$ for 2 min before the three components were thoroughly vortexed. Mixtures were then kept for $24 \mathrm{~h}$ in a stability oven set up at $25^{\circ} \mathrm{C}$ for equilibrium.

Table 1: Selected lipid formulations used to investigate the effect of oil composite on the solubility of DMY and the fate of drug after dispersion

\begin{tabular}{|c|c|c|c|c|c|}
\hline \multirow[t]{2}{*}{ Lipid formulation } & \multicolumn{5}{|c|}{ Excipients (\%w/w) } \\
\hline & Miglyol 812 & Imwitor 988 & Tagat T0 & Cremophor RH40, EL, or Crillet 4 & PEG 400 \\
\hline Type I & 70 & 0 & 30 & & 0 \\
\hline Type II & 49 & 21 & 30 & 0 & 0 \\
\hline Type IIIA & 42,49 , or 49 & 28,21 , or 21 & 0 & 30 & 0 \\
\hline Type IIIB & 0 & 30 & 0 & 30 & 40 \\
\hline
\end{tabular}

\section{The solubility of the model drug in lipid formulations}

The model drug (Dimethyl Yellow) was added in excessl\$) to either lipid excipients or to lipid formulations. Lipid suspensions were then vortexed for $3 \mathrm{~min}$ and then stored for $24 \mathrm{~h}$ in a controlled temperature oven at $25^{\circ} \mathrm{C}$ to reach equilibrium (samples were vortexed in between). Oil suspensions were centrifuged at maximum speed $(13000 \mathrm{~g})$ for $10 \mathrm{~min}$. The clear saturated oil solution was then removed and assayed analytically by UV spectrophotometry using a double beam instrument (Perkin-Elmer Lamboda 7, UV/VIS Spectrophotometer) at $\lambda$ max of $407 \mathrm{~nm}$.

\section{Preparation of the emulsification media (FaSSIF and FeSSIF)}

FaSSIF and FeSSIF were prepared by first making the micellar solution which consists of bile salt (Na Taurcholate) and Lecithin (L$\alpha$ phosphatidylcholin) at a ratio of 3:1. To prepare the mixed micellar solution appropriate quantities of bile salt $(15 \mathrm{mmol})$ and lecithin ( $3.75 \mathrm{mmol}$ ) were dissolved in $20 \mathrm{ml}$ of solvent consisted of methanol and chloroform at a ratio of $2: 3$. The resultant clear solution was placed in a $500 \mathrm{ml}$ Büchi flask and the solvent drawn off using rotary evaporator (Büchi Rotavapor R110). The flask was then attached to a vacuum line overnight to ensure the removal of any remaining chloroform. The clear film of bile salt and lecithin formed on the flask surface was subsequently dissolved in the appropriate buffer, see table 2 [19].

\section{Self-emulsification of oil systems containing dissolved DMY}

Lipid systems containing $4 \%$ of the model drug were emulsified using $1 \mathrm{gm}$ of the lipidic mixture in $100 \mathrm{ml}$ of different emulsification media; water, FaSSIF or FeSSIF. Dispersions were emulsified at $37^{\circ} \mathrm{C}$ for $15 \mathrm{~min}$ by gentle agitation at 100 oscillations/minute. The solubility of Dimethyl Yellow in the aqueous dispersions was monitored with time. Measurements were performed in triplicate values are expressed as mean values of all data \pm standard error.

Table 2: Composition of two physiological media used to simulate fasted state and fed state intestinal conditions

\begin{tabular}{llll}
\hline $\begin{array}{l}\text { Fasted state simulated intestinal } \\
\text { fluid (FaSSIF) }\end{array}$ & & $\begin{array}{l}\text { Fed state simulated intestinal } \\
\text { fluid (FeSSIF) }\end{array}$ \\
\hline $\mathrm{pH}$ & 6.5 & $\mathrm{pH}$ & 5 \\
Osmolarity & $270 \pm 10 \mathrm{~m} \mathrm{Osmol}$ & Osmolarity & $635 \pm 10 \mathrm{~m} \mathrm{Osmol}$ \\
Na Taurcholate & $3 \mathrm{mmol}$ & Na Taurcholate & $15 \mathrm{mmol}$ \\
Lecithin & $0.75 \mathrm{mmol}$ & Lecithin & $3.75 \mathrm{mmol}$ \\
$\mathrm{KH}_{2} \mathrm{PO}_{4}$ & $3.9 \mathrm{~g}$ & Acetic Acid & \\
$\mathrm{KCL}$ & $7.7 \mathrm{~g}$ & KCL & $8.65 \mathrm{~g}$ \\
$\mathrm{NaOH}$ & $\mathrm{qs} \mathrm{pH} 6.5$ & $\mathrm{NaOH}$ & $15.2 \mathrm{~g}$ \\
$\mathrm{Mili1} \mathrm{Q}$ water & $\mathrm{qs} 1$ liter & Mili Q water & $\mathrm{qs} \mathrm{pH} \mathrm{5}$ \\
\hline
\end{tabular}

\section{Particle size analysis}

Quasi-elastic light scattering (QELS, Malvern model LO-C photon correlation spectrometer) was used to analyze samples of submicron dispersions.

\section{RESULTS AND DISCUSSION}

\section{The solubility of dimethyl yellow (DMY in the lipid matrix)}

One commonly used approach to improve absorption is to solubilise the drug in a water-soluble cosolvent, such as polyethylene glycol which may have sufficient solvent capacity for drug administration as a unit dose. Yet, it is likely that the drug will crystallize on dilution of the cosolvent in the lumen of the gut. Unlike water-miscible cosolvent systems, lipids due to their immiscibility with water can maintain poorly soluble drugs in solution. However, when oil systems cannot provide sufficient solvent capacity for the drug in the unit dosage form, water-miscible cosolvents can be included in the pre-concentrate formulation. Therefore, verification of drug solubilization in the excipient matrix with a suitable assay for the drug is a crucial pre-formulation step that should be undertaken to assess the viability of any lipid system.

The solubility of DMY (a lipophilic model drug) in various watermiscible cosolvents and also in different types of lipid-based formulations is depicted in fig. 1. Maximum solubilization of DMY 
was obtained in Glycofurol followed by Transcutol. An approximately 2.5 fold decrease in the DMY solubility was observed in Polyethylene glycol 400 system vis-à-vis Glycofurol. The solubility of DMY, on the other hand, was the lowest in the case of Propylene glycol system. However, the solubility of DMY in various lipid formulae representing Type I, II, IIIA and IIIB (see, table 1 for composition) or in Miglyol 812 (source of triglycerides) was $\approx 40$ $\mathrm{mg} / \mathrm{g}$. Whereas, DMY solubility measured $20 \mathrm{mg} / \mathrm{g}$ in the case of more polar oil; Imwitor 988 .

Considering the cosolvent solubilization power $(\sigma)$ concept in the log-linear model proposed by Yalkowsky and coworkers [20-22], we can retrospectively work out $\sigma$ for DMY in the various cosolvent systems which were used in this study. The log-linear model describes an exponential increase in the solubility of non-polar drugs with a linear increase in cosolvent concentration. This is presented in the following equation:

$$
\log S_{\text {tot }}=\log S_{w}+\sigma X_{f_{c}} \text { (Eq. 1) }
$$

Where $S_{\text {tot }}$ is the total solubility of the drug in the cosolvent-water mixture, $\mathrm{S}_{w}$ is its water solubility, $\sigma$ is the cosolvent solubilization power for the particular cosolvent-solute system, and $\mathrm{f}_{\mathrm{c}}$ is the volume fraction of cosolvent in the aqueous mixture. For this study $\sigma$ was obtained from the $\log \left(S_{\text {tot }} / \mathrm{S}_{\mathrm{w}}\right)$ considering $\mathrm{f}_{\mathrm{c}}=1$ as no water was included in the cosolvent system. Table 3 lists the cosolvent solubilization power $(\sigma)$ obtained from Eq 1 and the partition coefficient $(\log \mathrm{P})$ of the considered cosolvent systems. The $\log \mathrm{P}$ values were obtained by means of the ClogP software package. The log $\mathrm{P}$ denotes the polarity of the cosolvent system. Table 3 suggests that the higher the $\log \mathrm{P}$ of the cosolvent, the lower the polarity and hence the higher the solubilization power. The following order is observed: $\log \mathrm{P}_{\text {Glycofurol }}>\log \mathrm{P}_{\text {Transcutol }}>\log \mathrm{P}_{\mathrm{PEG} 400}>\log \mathrm{PG}$. The least polar cosolvent, Glycofurol, has the maximum solubilization power for DMY $(\sigma=4.8)$ and the highest polar cosolvent, $\mathrm{PG}$, has the lowest value $(\sigma=3.19)$.

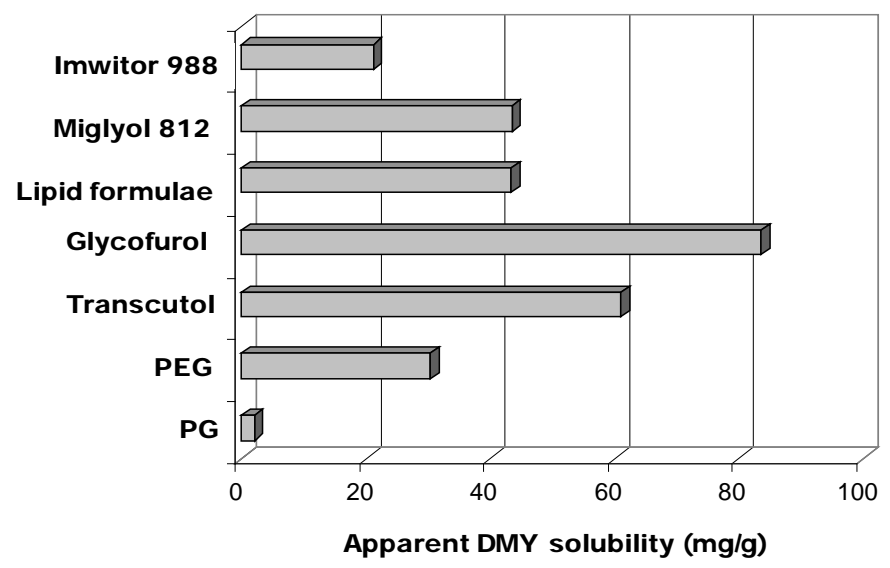

Fig. 1: Apparent solubility of DMY in various hydrophilic cosolvents, lipid excipients and oil formulae representing lipid class systems type II, IIIA and IIIB; see table 1 for. The drug was added in excess to these systems and left to equilibrate at $25^{\circ} \mathrm{C}$ for $24 \mathrm{~h}$

Table 3: The effect of cosolvent polarity on the cosolvent solubilization power ( $\sigma$ ) for DMY in PG, PEG 400, Transcutol and Glycofurol (sorted by increasing $\log \mathrm{P}$ )

\begin{tabular}{llll}
\hline Cosolvent system & Cosolvent $\log \mathbf{P}$ & Solubilization power $(\boldsymbol{\sigma})$ & Solubility of DMY mg/g \\
\hline Propylene glycol & -0.92 & 3.19 & $2.06 \pm 0.002$ \\
Polyethylene glycol 400 & -0.88 & 4.36 & $30.46 \pm 0.42$ \\
Transcutol & -0.15 & 4.66 & $60.99 \pm 1.54$ \\
Glycofurol & -0.04 & 4.80 & $83.53 \pm 1.69$ \\
\hline
\end{tabular}

*Each value represents mean $\pm S$. $D(n=3)$.

This is expected since less polar cosolvents can reduce selfassociation than polar cosolvents as they have a better affinity for the non-polar compounds. It is worth noting here that, Polyethylene glycol 400 has higher $\sigma$ value than Propylene glycol albeit they both have close $\log \mathrm{P}$ values. This might be due to the large area of interaction with the DMY in the case of PEG 400 due to the relatively high number of polyoxyethylene residues (an average of 9 residues per molecule). Furthermore, identical solubilization power of 4.5 was found for these lipid systems, yet, with a lower value in the case of Imwitor $988(\sigma=4.2)$ as it is a more polar oil due to the monoglyceride content. On the other hand, the inclusion of PEG 400 in the oil mixture to formulate Type IIIB did not, however, enhance the solubility of DMY as PEG 400 has a relatively low solubilization power. If a high DMY solubility is required, cosolvents with high solubilization power such as Glycofurol or Transcutol can be used.

Physicochemical parameters influencing the solvency of dispersions formed by various lipid-based formulations

Hydrophilicity of the lipid-vehicle: oil formulations Vs cosolvent systems

Studies generally, in the field of lipid-based formulations, focus on investigating the effect of the drug on the physicochemical characteristics of the resultant dispersions in particular, emulsion droplet size as it is crucial for the enhanced bioavailability. Also, equilibrium phase behavior is being studied in the presence of the drug, especially if the drug has surface activity, in order to establish an empirical link between these effects incurred by the inclusion of a particular drug and emulsification [23]. Nonetheless, there have not been many reports which outline how the physicochemical properties of emulsions formed by SEDDS can influence the fate of dissolved drug after dispersion. We are investigating the effect of the solvent capacity of these dispersions on the solubilization behavior of the drug and how factors such as oil-cosurfactant ratio, type of surfactant and the inclusion of various cosolvents can influence this behavior in an attempt to circumvent the precipitation of drug.

Photos presented in fig. 2 and 3 outline the effect of various types of either lipid-based formulations or cosolvent systems on the precipitation of drug after dispersion of the formulation. Fig. 2 shows the aqueous dispersions of oil formulations in the presence of approximately $40 \mathrm{mg} / \mathrm{g}$ DMY representing Type II lipid-class system (bottles A and B), and Type IIIA (bottles C, D, and E), see table 1 for composition. In Type II lipid systems, the pre-concentrate mixture, which is composed of water-insoluble materials, was selected to from either a self-emulsified dispersion of particle size more than 
$250 \mathrm{~nm}$ or a microemulsion of particle size $<100 \mathrm{~nm}$, bottles A and B, respectively. On the other hand, various types of hydrophilic surfactants Cremophor RH40, EL or Crillet 4 were included in Type IIIA systems, bottles, C, D or E, respectively. Visual assessment of dispersions depicted in fig. 2 did not reveal any crystallization of DMY in the first $24 \mathrm{~h}$. Nonetheless, dispersions which formed fine emulsions (bottles B and C) were subjected to further analytical assessment for several days in order to study the solubilization behavior of DMY with time in these systems. Furthermore, an instant crystallization of DMY occurred from the emulsification of either Imwitor 988/RH40-PEG 400 (30/30/40) system, an archetypal example of Type IIIB hydrophilic lipid formulations, or cosolvent-based systems (Glycofurol and Transcutol), see photo in fig. 3).

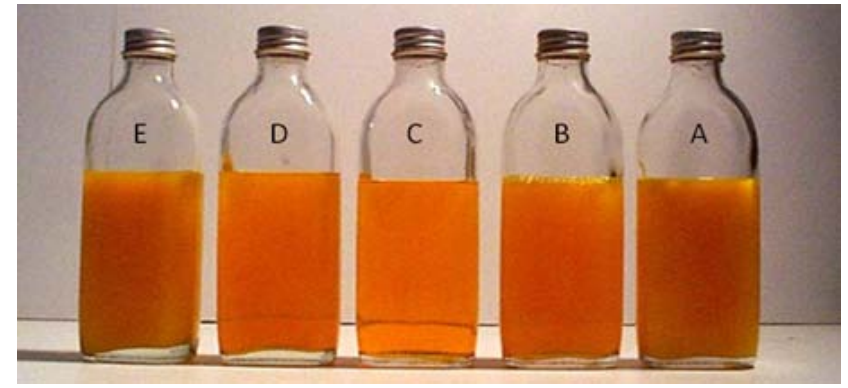

Fig. 2: Photograph of the effect of various lipid systems on the solubilization of DMY after the dispersion of the formulation. Bottles A to E represent dispersions of formulations representing type II and type IIIA lipid class system. $1 \mathrm{~g}$ of each formulation containing approximately $40 \mathrm{mg}$ DMY was allowed to emulsify in $100 \mathrm{ml}$ water at $37^{\circ} \mathrm{C}$ for $15 \mathrm{~min}$. Dispersions were assessed visually and analytically for detecting any drug precipitation due to loss of solvent capacity

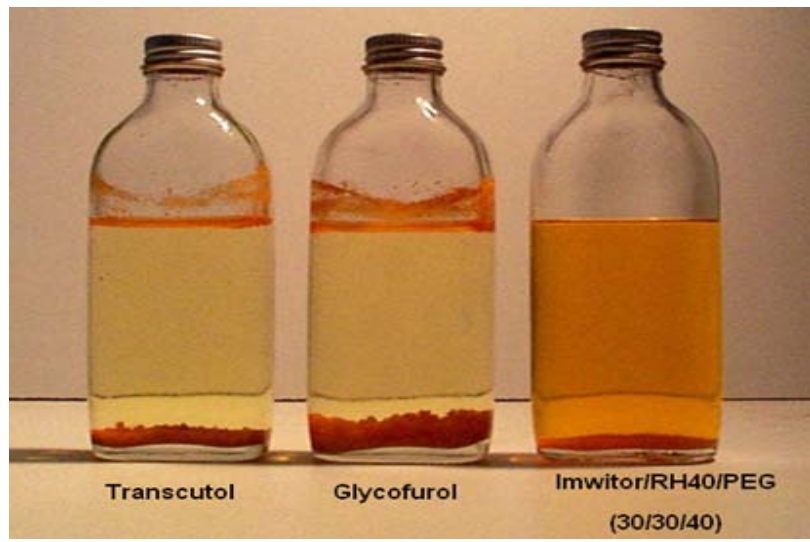

Fig. 3: Photograph of the effect of either Type IIIB lipid class formulation (Imwitor 988/Cremophor RH40-PEG 400) or water-miscible cosolvent based systems (Glycofurol and Transcutol) on the solubilization of DMY after the dispersion of the formulation. $1 \mathrm{~g}$ of each formulation was emulsified in the presence of DMY in $100 \mathrm{ml}$ water at $37^{\circ} \mathrm{C}$ for $15 \mathrm{~min}$

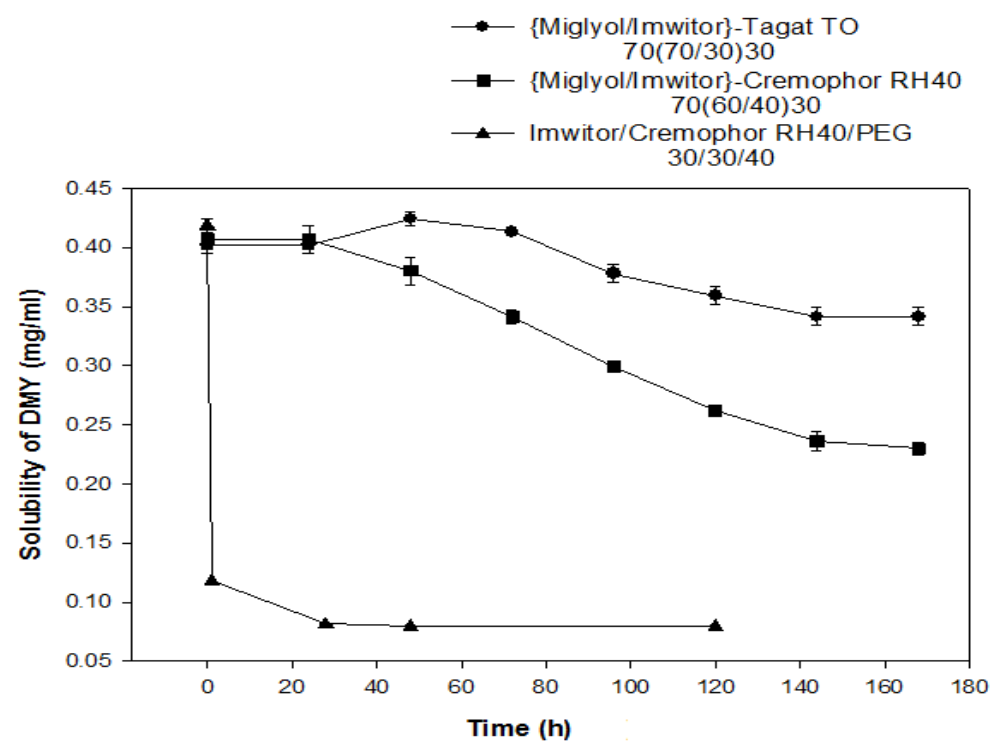

Fig. 4: Effect of hydrophilicity of the oil vehicle on the solubilization profiles of DMY with time. Lipid formulations representing Type II

(O), IIIA ( $\square$ and IIIB ( $\triangle$ and containing around $40 \mathrm{mg} / \mathrm{g}$ DMY were allowed to emulsify (1g) in $100 \mathrm{ml}$ of water at $37^{\circ} \mathrm{C}$ for $15 \mathrm{~min}$. Dispersions were kept for various periods of time for probing drug precipitation. Bars represent standard errors $(n=3)$ 


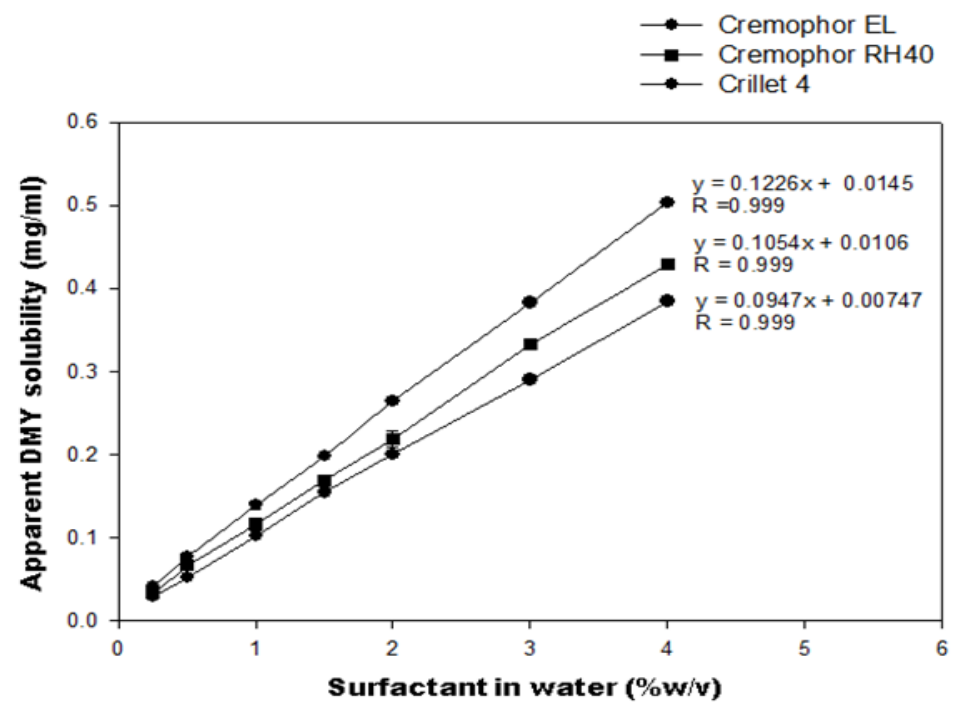

Fig. 5: Solubility of DMY in various surfactant-water mixtures as a function of surfactant concentration

Type IIIB formulations generally produce the finest dispersions because of their high content of water-soluble solubilizing agents, viz. they contain more than $60 \%$ hydrophilic surfactant and/or water-miscible cosolvents [3]. Hence, the water-soluble components tend to diffuse away from the oil during dispersion and become dissolved in the aqueous phase. The result of this separation, which may be the driving force for emulsification by 'diffusion and stranding', is likely to be a loss of solvent capacity [17]. As a consequence, the drug is partially precipitated when the formulation disperses. This was also evident from the water-miscible cosolvent systems glycofurol and transcutol whereby, spontaneous crystallization of DMY occurred after dilution in the aqueous phase [24-25]. An example of a co-solvent formulation is vinorelbine (Navelbine ${ }^{\circledR}$ ) [26]. The extent of precipitation will depend on the hydrophilicity of the lipid system, the particle size of the resultant dispersion, the contribution of the hydrophilic material (hydrophilic surfactant and/or cosolvent) to the solubilization of the drug within the formulation and the $\log \mathrm{P}$ of the drug.

Fig. 4 displays the effect of hydrophilicity of the lipid matrix, as controlled by the type of excipients included in the system, on the solubilization behaviour of DMY. The \{Miglyol 812/Imwitor 988\}Tagat TO at ratio of $(70(70 / 30) 30)$ is a pre-microemulsion Type II lipid mixture [17]. This type of formulation is composed of waterinsoluble materials i.e. a hydrophobic system. Hence, it is expected to retain its solvent capacity for the drug after dispersion. It is evident from the emulsification of this system in the presence of DMY that the solvency of the resultant dispersion was able to keep the drug in the super-saturated state for up to $3 \mathrm{~d}$ after the emulsification event. However, at equilibrium which can take up to five days, only $6 \mathrm{mg}$ of DMY (15\%) out of an initial dose of approximately $40 \mathrm{mg}$ came out of solution. This limited amount of crystallized material after $5 \mathrm{~d}$ might be due to the fact that the system forms fine dispersion on emulsification of less than $100 \mathrm{~nm}$ so oil droplets which contain the dissolved drug will be highly exposed to the aqueous phase due to large surface area of contact and thus minimal crystallization has occurred. This process, however, might take long time to reach equilibrium as it was demonstrated. On the other hand, the self-emulsified type II class lipid-system which forms dispersion with particle size of $>250 \mathrm{~nm}$ such as Miglyol 812/Tagat TO (bottle A, fig. 2) had shown no indication for DMY crystallization. This might be attributable to the relatively large droplet size whereby, DMY will be sequestered within the oil phase. Hence, there will be virtually minimal interaction between the non-polar compound and the water's hydrogen network which will reduce the ability of water to induce crystallization due to self-association. On the other hand, for Type IIIA lipid system, \{Miglyol 812/Imwitor 988\}-Cremophor RH40 at a ratio of $70(60 / 40) 30$, DMY was able to remain in a super-saturated state up to $24 \mathrm{~h}$ after emulsification (fig. 4). The crystallization of DMY from this system was also found to take up to $5 \mathrm{~d}$ to reach equilibrium.
Unlike the limited crystallization which occurred at equilibrium from the self-micro-emulsified type II lipid-system (fig. 4), approximately $42 \%$ of the initial DMY dose ( $40 \mathrm{mg}$ ) precipitated out from the Type IIIA system. This might by due to two factors, one of which is the amount of DMY which the hydrophilic surfactant (Cremophor RH40) was contributing to its solubilization within the formulation.

The amount of solubilized DMY within the pre-concentrate mixture which is contributed by the hydrophilic surfactant can be calculated using the following equation:

$$
S_{\text {total }}=F_{A} X S_{A}+F_{B} X S_{B}+F_{C} X S_{C}(\text { Eq. 2) }
$$

where $S$ total is the total solubility of DMY in the lipid mixture, $F_{A}, F_{B}$ and $\mathrm{F}_{C}$; mole fraction of each lipid excipient in the oil system, and $\mathrm{S}_{\mathrm{A}}, \mathrm{S}_{\mathrm{B}}$ and $\mathrm{S}_{\mathrm{C}}$; the respective DMY solubility in each constituent.

In the case of $\{$ Miglyol 812/Imwitor 988\}-Cremophor RH40 system at ratio of $70(60 / 40) 30$, an amount of approximately $19 \mathrm{mg}$ of DMY is solubilized within $1 \mathrm{~g}$ of the oil mixture due to the contribution of Cremophor RH40. On emulsification of $1 \mathrm{~g}$ formulation containing around $40 \mathrm{mg}$ DMY in $100 \mathrm{ml}$ of water, Cremophor RH40 which has a concentration of $30 \% \mathrm{w} / \mathrm{w}$ in the pre-concentrate, parts from the oil during dispersion and becomes diluted in the aqueous phase making a final concentration of $0.3 \% \mathrm{w} / \mathrm{v}$. By applying the linear regression equation for Cremophor RH40 system presented in fig. 5 which relates the increase of DMY solubility in Cremophor RH40water system as a function of surfactant concentration, we can work out the amount of DMY that the surfactant can maintain in solution after the dispersion of the formulation. The dissolved surfactant in the aqueous phase after dispersion can maintain an amount of around $5 \mathrm{mg}$ of DMY in solution out of the $19 \mathrm{mg}$ which Cremophor RH40 is responsible for in the initial pre-concentrate dose. Therefore, approximately $14 \mathrm{mg}$ of DMY $(\approx 35 \%$ of initial dose) will tend to come out of solution at equilibrium as a result of diffusion of the hydrophilic surfactant into the aqueous phase after dispersion. Another important factor which might further initiate crystallization of DMY from Type IIIA lipid system is the large surface available for contact with water due to the formation of very fine oil particles after dispersion. In the case of the very hydrophilic Type IIIB lipid system, the risk of precipitation is greater as the formulation contains a higher proportion of hydrophilic components (hydrophilic surfactants and/or water-soluble cosolvents). This is demonstrated by the instant crystallization of DMY from the Imwitor 988/Cremophor RH40-PEG 400 Type IIIB lipid system depicted in fig. 4 Almost $70 \%$ of an initial DMY dose of $4 \quad 0 \mathrm{mg}$ came out of solution in the first hour after the dispersion of the formulation. The amount of DMY that is dissolved within the lipid mixture due to the contribution of Cremophor RH40 or PEG 400 is around 23 or $12 \mathrm{mg}$, respectively. This means that the hydrophilic components in the Imwitor 988/Cremophor RH40-PEG 400 system are responsible for 
solubilizing approximately $35 \mathrm{mg}$ of DMY in the lipid matrix. Cremophor RH40 and PEG 400 can keep up to only $5 \mathrm{mg}$ of DMY in solution after the dispersion of the formulation as they tend to lose their solvent capacity on dilution with water. Therefore, $30 \mathrm{mg}$ of DMY, which is almost equivalent to $75 \%$ of DMY initial dose, is expected to come out of solution after the emulsification. This is, however, in full agreement with the solubilization profile of DMY from this system which is depicted in fig. 4.

The partition coefficient $(\log \mathrm{P})$ of the drug is an important physicochemical factor which influences the formulation design for lipid systems. Generally, drugs with $\log \mathrm{P}$ values $<2$ are the most difficult drugs as they have limited solubility in both water and lipid. Therefore, it is unlikely that lipid formulation will be of value for such compounds [3]. On the other hand, more hydrophobic drugs may have good permeability to lipid membranes but dissolve very slowly in the lumen of the gut. Hydrophobic drugs with intermediate $\log$ P [2-4] may benefit the most from the formulation design of lipid-based systems. Hydrophilic surfactants and/or water-soluble cosolvents can be blended with the lipid systems to increase the solvent capacity of the formulation for molecules with intermediate log P. Yet, the choice between the different types of lipid-based formulations (Type I, II or III) has to be made considering the risk of drug precipitation and also the desirability of rapid absorption. Furthermore, drugs with $\log \mathrm{P}>2$ are likely to be solubilized by bile salt micelles during digestion which in effect might prevent the drug from precipitation in the gut. Drugs with $\log P>5$ can also benefit from the reformulation with lipid systems yet digestion by lipolysis will be crucial for the absorption of these drugs. The natural process of digestion offers the possibility that highly lipophilic drugs with $\log P$ values greater than 5 and triglyceride solubility of at least $50 \mathrm{mg} / \mathrm{ml}$ are preferentially transported via lymphatic route [27-28]. Lymphatic transport may be enhanced by lipid-based formulations yet the enhancement depends on the nature of the vehicle. According to a study by Porter et al. [29], the rate and extent of lymphatic transport of a highly lipophilic drug was of the following rank order; micellar>emulsion>lipid solution.

\section{Effect of oil-cosurfactant ratio on the solvency of dispersions}

As it was demonstrated by Hasan et al. [17-18], oil-cosurfactant ratio in the pre-concentrate mixture is an important parameter which determines the droplet size of the resultant emulsion. Nevertheless, in the case of hydrophilic surfactants with high solubilization capacity such as Cremophor RH 40 or EL, oil-cosurfactant ratio becomes also a crucial factor in affecting the kinetics of drug crystallization after the dispersion of the oil-system. This becomes more evident in the case of high hydrophilic lipid formulations and in particular, when water-miscible cosolvent is included in the system. By increasing the oil fraction in oilcosurfactant blend, we increase the non-polar hydrocarbon regions which interfere with water's hydrogen bonding network. As a result, water's ability to "squeeze out" non-polar compounds is reduced and hence the rate of crystallization is retarded.

Fig. 6 to 9 display the effect of oil-cosurfactant ratio in Miglyol 812/Imwitor 988-Cremophor RH40 system containing increasing concentration of PEG 400 on the solubilization behaviour of DMY after aqueous dispersion. For Miglyol 812-Imwitor 988 at ratios of 1:9 and 3:7 without PEG 400, gradual loss of DMY solubility was observed (fig. 6 and 7, respectively). Yet, in the case of 5:5 system (without PEG 400), DMY was maintained in supersaturated state for up to $24 \mathrm{~h}$ from the initial event of emulsification (fig. 8). However, the inclusion of PEG 400 at only $10-20 \%(w / w)$ in these systems accelerated DMY precipitation. There had been progressive drop in the solubility of DMY due to drug crystallization as the concentration of PEG 400 in the formulation was increased. The dissolved amount of DMY within the pre-concentrate mixture is enhanced as more cosolvent is used in the system. On dispersion of the formulation and due to loss of solvent capacity as hydrophilic components diffuse into the aqueous phase, crystallization of DMY occurs depending on the extent the cosolvent was contributing to its solubilization within the oil mixture, see table 4 .

For Miglyol 812-Imwitor 988 (1:9) or (3:9) systems at $10 \%$ or $20 \%$ PEG 400, more than half of the drug precipitated within the first $6 \mathrm{~h}$ (table 4), which suggests that precipitation from SEDDS containing a cosolvent is likely to occur within the lumen of the gut. In contrast, when the Miglyol 812 content was further increased in the oil blend as in (5:5) system, only 10 and $30 \%$ of the DMY dose came out of solution in the case of inclusion either 10 or $20 \%$ PEG, respectively (table 4). Therefore, by increasing the weight fraction of Miglyol 812 in the oil-cosurfactant blend, the solvency of the lipid system to maintain the drug in solution after the dispersion of the formulation is enhanced and consequently, precipitation is retarded, see fig. 9. Furthermore, the effect of including water-miscible cosolvent in Type II self-micro-emulsified lipid system, which is composed of water insoluble materials, on the crystallization of DMY after emulsification was also investigated and presented in fig. 10. The solubilization profiles of DMY observed from Miglyol 812/Imwitor 988 (7:3)-Tagat T0 system with and without any cosolvent have shown similar trend to the Miglyol 812/Imwitor 988 (5:5)Cremophor RH40 system (fig. 10 and 8, respectively). The inclusion of $10 \%$ PEG in the system did not influence the solubilization behaviour of DMY yet, around $25 \%$ of the drug precipitated after $6 \mathrm{~h}$ from dispersion when 20\% PEG was included in the pre-concentrate (fig. 10). The migration of the cosolvent into the aqueous phase and large surface area of contact with water due to the formation of fine dispersion are the two main factors which affect the crystallization of DMY in this system. Fig. 11 displays the effect of inclusion various cosolvents in the Miglyol 812/Imwitor 988 (5:5)-Cremophor RH40 system on the solubilization behaviour of DMY after the dispersion of the formulation. The solubilization profiles of DMY were comparable for oil systems containing either PEG 400 or Ethanol. On the other hand, in the case of using transcutol or glycofurol, almost equivalent profiles were also obtained yet with relatively high crystallization rate vis-à-vis formulations containing PEG 400 or Ethanol. This is attributed to the high solubilization power of these cosolvents as they are able to dissolve more DMY within the lipid formulation which tend to crystallize on dispersion.

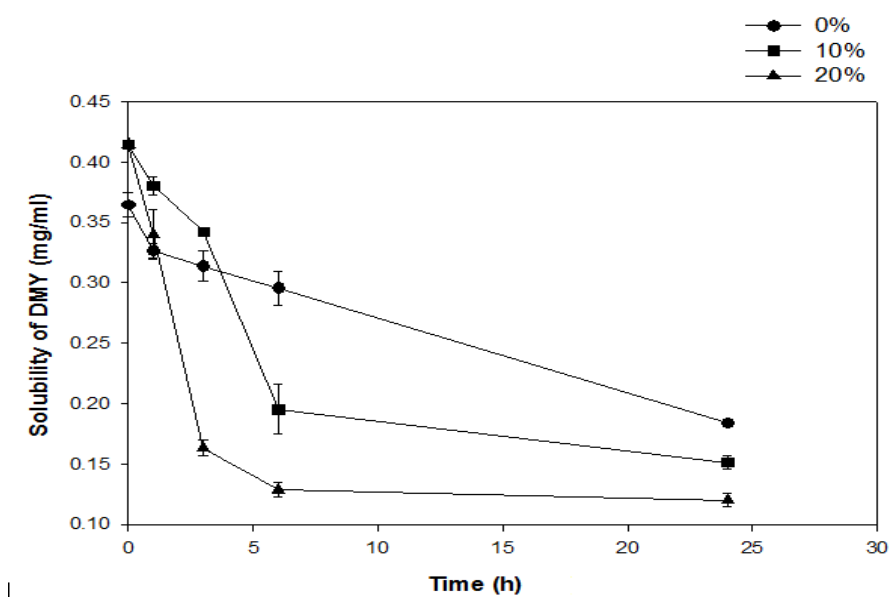

Fig. 6: Effect of inclusion increasing concentration of PEG 400 in the miglyol 812/Imwitor 988-cremophor RH40 \{70(10/90)30\} system on the solubilization behaviour of DMY after aqueous dispersion. Bars represent standard errors $(n=3)$ 


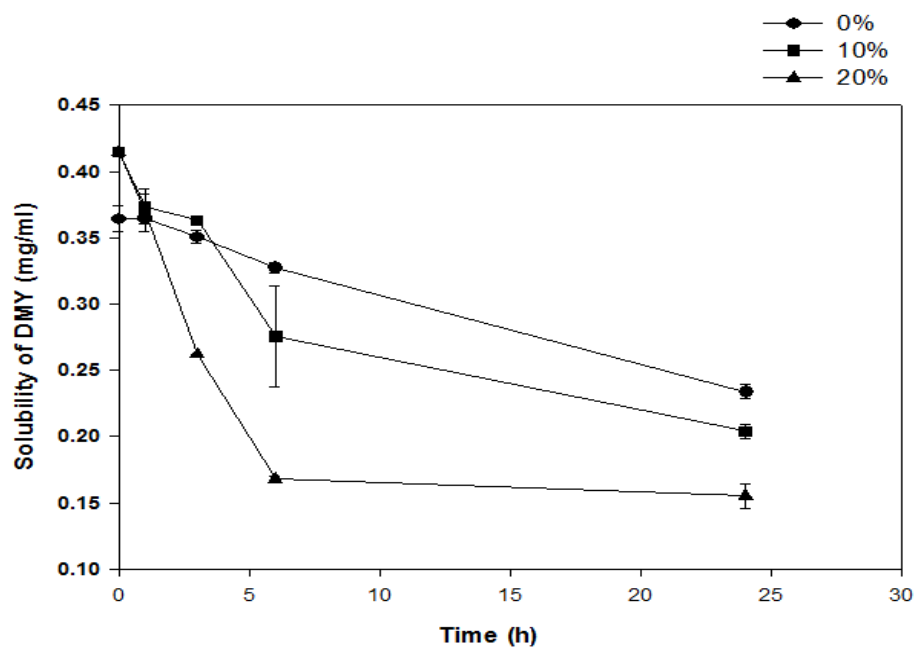

Fig. 7: Effect of inclusion increasing concentration of PEG 400 in the Miglyol 812/Imwitor 988-cremophor RH40 \{70(30/70)30\} system on the solubilization behaviour of DMY after dispersion in water. Bars represent standard errors $(n=3)$

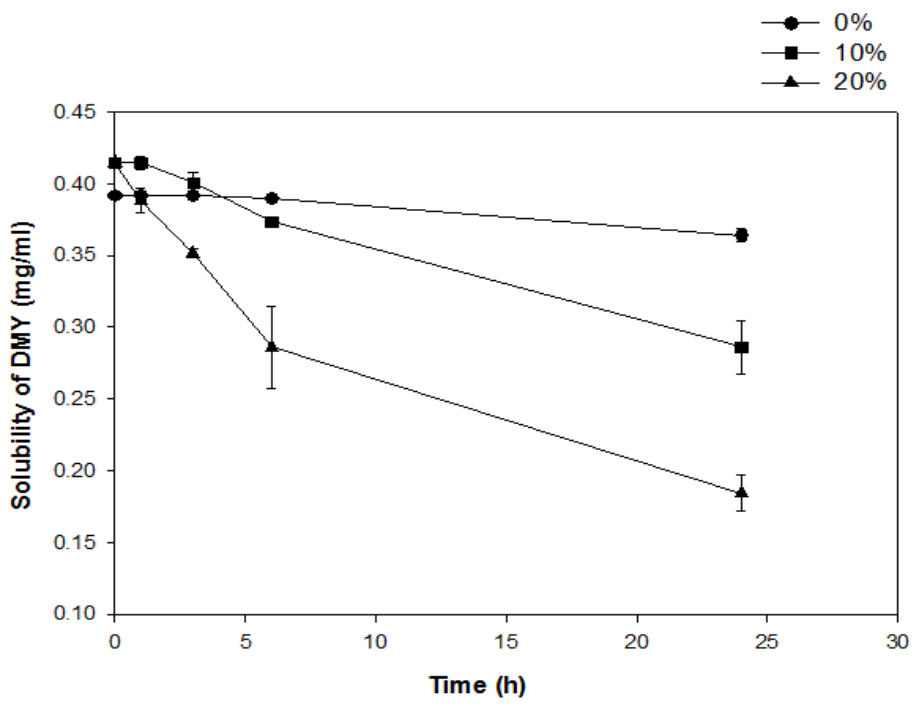

Fig. 8: Effect of inclusion increasing concentration of PEG 400 in the Miglyol 812/Imwitor 988-cremophor RH40 \{70(50/50)30\} system on the solubilization behaviour of DMY after aqueous dispersion. Bars represent standard errors $(n=3)$

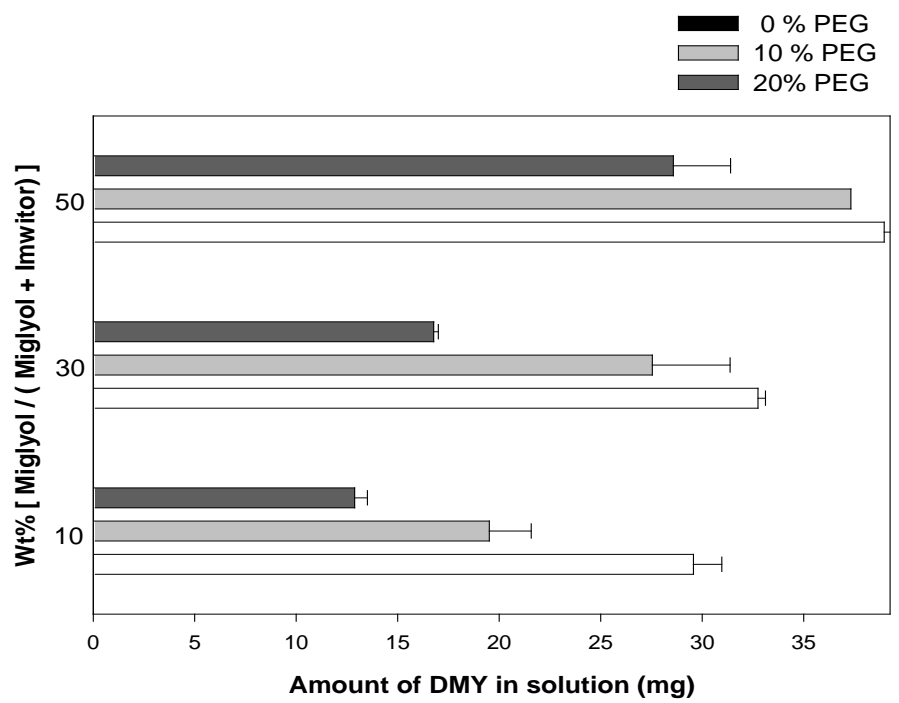

Fig. 9: The effect of oil-cosurfactant ratio in the pre-microemulsion concentrate of Miglyol 812/Imwitor 988-Cremophor RH40 at increasing concentration of PEG 400 on the solubilization of DMY after $6 \mathrm{~h}$ from the emulsification event. 1g of each formulation containing approximately $40 \mathrm{mg}$ DMY was allowed to emulsify in $100 \mathrm{ml}$ water at $37^{\circ} \mathrm{C}$ for $15 \mathrm{~min}$ bars represent standard errors $(\mathrm{n}=3$ ) 
Table 4: The effect of oil-cosurfactant ratio on the crystallization of DMY from the Miglyol 812/Imwitor 988-cremophor system containing various percentages of PEG 400 (Miglyol 812 is used as oil, Imwitor 988 as a cosurfactant and cremophor RH 40 is the hydrophilic surfactant)

\begin{tabular}{llll}
\hline Oil: cosurfactant & $\mathbf{1 : 9}$ & $\mathbf{3 : 9}$ & $\mathbf{5 : 5}$ \\
\hline PEG 400 \% (w/w) & Amount of precipitated DMY after $\mathbf{6 h}$ from dispersion $\mathbf{( m g )}$ & \\
\hline 0 & $6.88 \pm 0.54$ & $3.72 \pm 0.61$ & $\approx 0$ \\
10 & $21.95 \pm 2.4$ & $13.94 \pm 3.44$ & $4.32 \pm 0.17$ \\
20 & $28.58 \pm 0.91$ & $24.54 \pm 0.53$ & $12.88 \pm 3.14$ \\
\hline
\end{tabular}

*Each value represents mean $\pm \mathrm{SD}(\mathrm{n}=3)$.

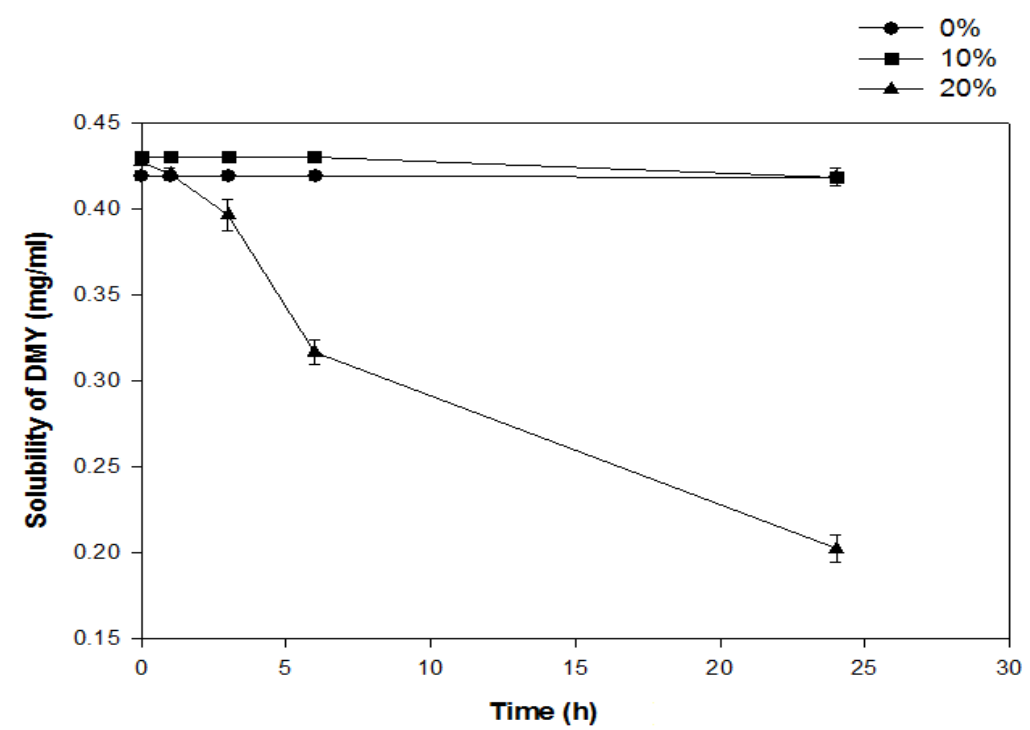

Fig. 10: Effect of inclusion increasing concentration of PEG 400 in type II self-micro-emulsified lipid system (Miglyol 812/Imwitor 988Tagat TO) on the solubilization behaviour of DMY after dispersion in water. Bars represent standard errors $(\mathrm{n}=3)$

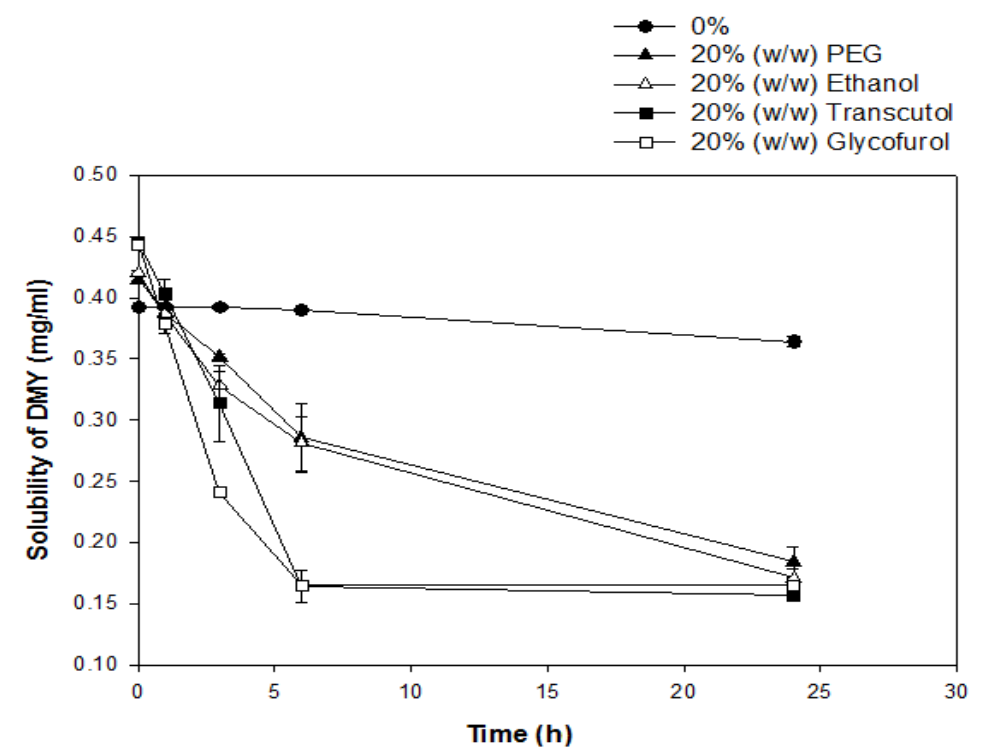

Fig. 11: Effect of inclusion water-soluble cosolvents with various solubilization powers ( $\sigma$ ) in the Miglyol 812/Imwitor 988 (5:5)Cremophor RH40 system on the solubilization profiles of DMY. Bars represent standard errors $(n=3)$

\section{Effect of the ionic strength of Emulsification media}

In order to establish better in vitro-in vivo correlations for the oral administration of poorly water-soluble drugs, the composition, volume and hydrodynamics of the contents in the gastrointestinal lumen following the administration of the dosage form need to be accurately simulated [30]. Four media have been developed to simulate composition of the gastric and intestinal contents before and after ingested meal. Simulated Gastric Fluid (SGF) is used to simulate fasted gastric conditions, homogenized long-life milk $(3.5 \%$ fat, $\mathrm{pH}$ 6.5) has been suggested to simulate fed state stomach, FaSSIF and FeSSIF to model fasted and fed state conditions in the small intestine, respectively.

In this study the effect of FaSSIF and FeSSIF media on the solubilization behaviour of DMY from Type II and Type III lipid class 
systems were investigated. In order to establish the importance of the bile salt-lecithin mixed micelles in the kinetics of drug crystallization from these systems, FaSSIF medium was prepared without bile salt and lecithin, see table 2 . This will give us an insight into the solubilization capacity of the endogenous surfactants and moreover will address the effect of electrolytes on the solvency of these lipid systems after dispersion.

Fig. 12 depicts the crystallization of DMY from Miglyol 812/Imwitor 988 (7:3)-Tagat TO system after the emulsification in water or FaSSIF medium without bile slats-lecithin mixed micelles. As this fig. shows, the emulsification of this system in FaSSIF accelerated the crystallization of DMY. At equilibrium which took up to $5 \mathrm{~d}$ to reach, around $15 \%$ of drug precipitated when the system was emulsified in water in comparison with $35 \%$ in the case of FaSSIF. The effect of electrolytes on the emulsification behaviour of this system without drug was thoroughly studied in by Hasan et al. [17]. Generally, electrolytes reduce the hydrophilicity of the non-ionic surfactants and hence they become more soluble in the oil phase. This will cause depression in the phase inversion temperature (PIT) of the system and eventually and phase separation. This effect will depend on the inclusion of high polar oil in the formulation like Imwitor 988 and moreover, the use of non-ionic surfactant with relatively low HLB such as Tagat TO. In this case, the reduction in the HLB of the surfactant incurred by the presence of electrolytes in the emulsification media will be sufficient to cause shift in its solubility from the aqueous to the oil phase which results in phase separation and eventually crystallization of the dissolved drug. However, for Miglyol 812/Imwitor 988 (7:3)-Tagat TO phase separation was not seen yet, there had been substantial increase in the droplet size due to the shift in Tagat To solubility. Nonetheless, total phase separation was observed after the dispersion in FaSSIF by including more Imwitor 988 in the oil blend as in the case of Miglyol 812/Imwitor 988 (6:4)-Tagat T0.

It is expected that the use of surfactant systems with high HLB value such as, Cremophor RH40 or EL will not be affected by the ionic strength of the emulsification media as the reduction in the surfactant HLB is not sufficient to induce phase separation. This was evident from the crystallization profiles of DMY from Miglyol 812/Imwitor 988 (5:5)Cremophor system depicted in fig. 13. Almost comparable trends were observed from the emulsification in water or FaSSIF. In both media more than $40 \%$ of DMY came out of solution at equilibrium, vis-à-vis $15 \%$ in the case of Miglyol 812/Imwitor 988 (7:3)-Tagat TO when emulsified in water (fig. 12). This is attributed to the fact that the former system contains a hydrophilic surfactant that tends to lose its solvent capacity after the dispersion.

As depicted in fig. 12 and 13 both formulations can take up to $5 \mathrm{~d}$ to reach equilibrium and that the drug can remain in a supersaturated state for up to $24 \mathrm{~h}$ after the initial emulsification event. It could be argued that such products are unlikely to cause precipitation of the drug before the drug is absorbed and moreover, super-saturation might enhance absorption by increasing the thermodynamic activity of the drug [3].

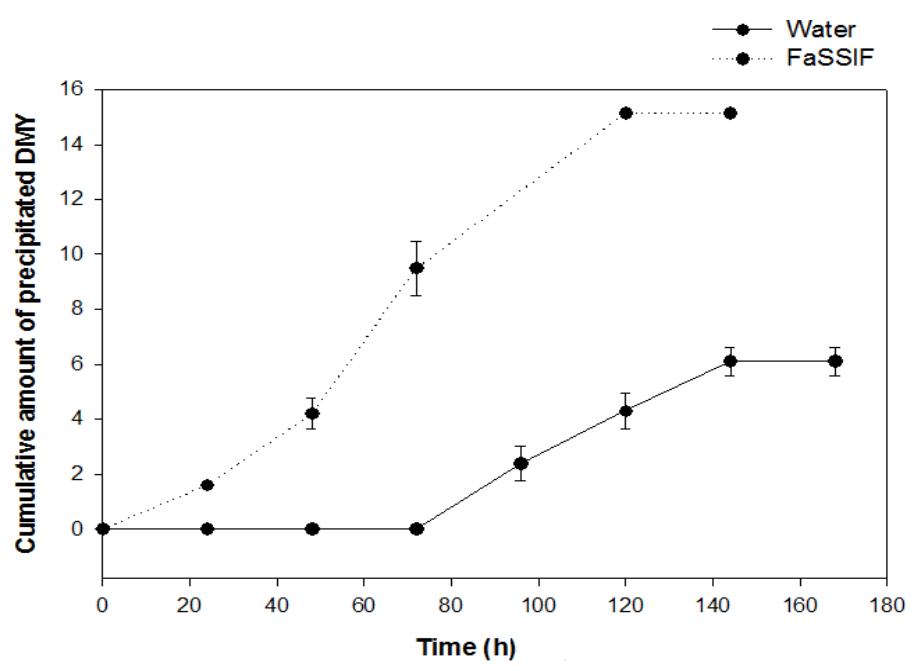

Fig. 12: Effect of ionic strength of the emulsification media on the crystallization of DMY as function of time from Type II self-microemulsified lipid system which contains water insoluble materials (Miglyol 812/Imwitor 988-Tagat T0 70(70:30)30). FaSSIF medium was used here without the bile salt-lecithin mixed micelles; see the text for further elaboration. Bars represent standard errors $(n=3)$

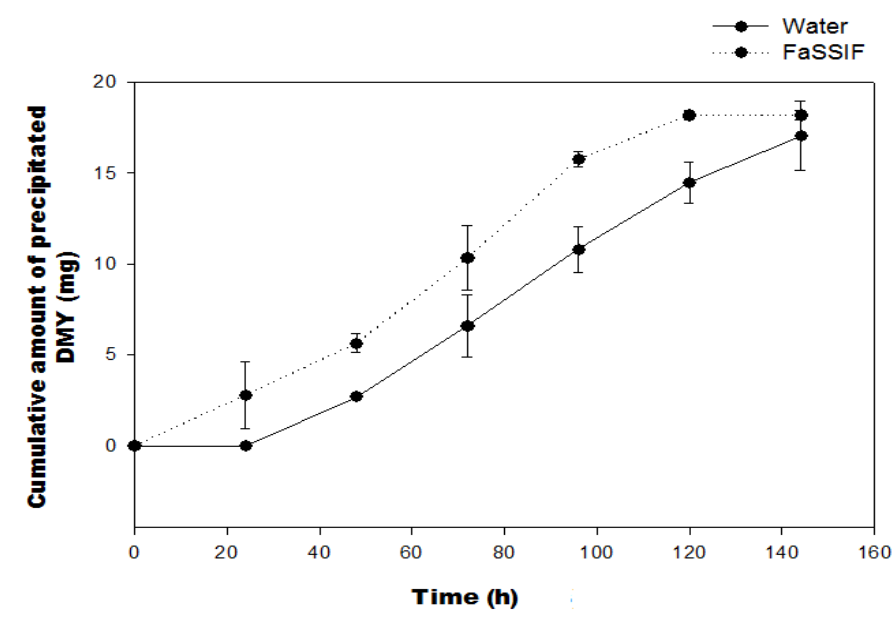

Fig. 13: Effect of ionic strength of the emulsification media on the crystallization of DMY as function of time from Type III self-microemulsified lipid system which contains a hydrophilic surfactant with relatively high HLB value (Miglyol 812/Imwitor 988-Cremophor RH40 70(50:50)30). Bars represent standard errors $(n=3)$ 


\section{The effect of bile salt-lecithin mixed micelles}

In the small intestine, drug solubility can be enhanced by the secretion of bile salts and other endogenous amphiphilic biliary components including lecithin and cholesterol. At concentrations higher than critical micelle concentration (CMC) these substances form mixed micelles which can enhance the solubilization capacity of the GI tract. Solubilization enhancement into simple bile salt micelles has been reported for many lipophilic compounds [31]. Up to 100 -fold increase in solubility has been observed upon addition of bile salts at physiological concentrations to aqueous media. Moreover, Dissolution rate for many lipophilic compounds was substantially improved in the presence of bile salts. Improve the wetting was the predominant mechanism for substances at log $P$ values in the range of 1-2. However, in the case of highly lipophilic compounds such as danazol (log P 4.53), the increase in powder dissolution rate was attributed to the solubilization enhancement [32].

On the other hand, bile salt-lecithin mixed micelles was found to further enhance the solubility of lipophilic compounds [33-35]. Addition of lecithin causes an increase in the molecular weight of micelles from 6000to 150,000 Dalton [36] and hence more molar volume could be included into the palisade layer of the micelle. The extent of solubilization was shown to be influenced by the ratio of bile salts to lecithin [37]. Nonetheless, at high mixed micelles concentration enormous increase of the micellar diameter occurs. Therefore, a consequence of drug solubilization within micelles is a decrease in the apparent diffusion coefficient [38], since the effective diffusivity will be that of the micelle rather than of the drug monomer.

Mithani et al. [39] studied the solubilization of a range of drugs by taurocholate solutions, and based on the drug log $\mathrm{P}$ and aqueous solubility developed good predictive estimates of the solubilization enhancement by bile salts. A linear correlation (Eq. 5) was observed between the logarithm of the solubilization ratio (SR), and log P.

$$
[\mathrm{SR}]=0.64 \mathrm{X} \log [\mathrm{P}]+2.09 \text { (Eq. 3) }
$$

Solubilization ratio (SR) can be defined as the ratio between the solubilizing capacity of bile salt micelles (SCbs) measured as moles $\mathrm{drug} / \mathrm{mole}$ taurocholate and the solubilization capacity in water (SCaq). According to this model, the process of solubilization is entirely driven by the hydrophobicity of the drug. Yet, in addition to partitioning behaviour other factors such as, the drug molecular weight or shape, and the affinity for bile salt micelles can also play a role.

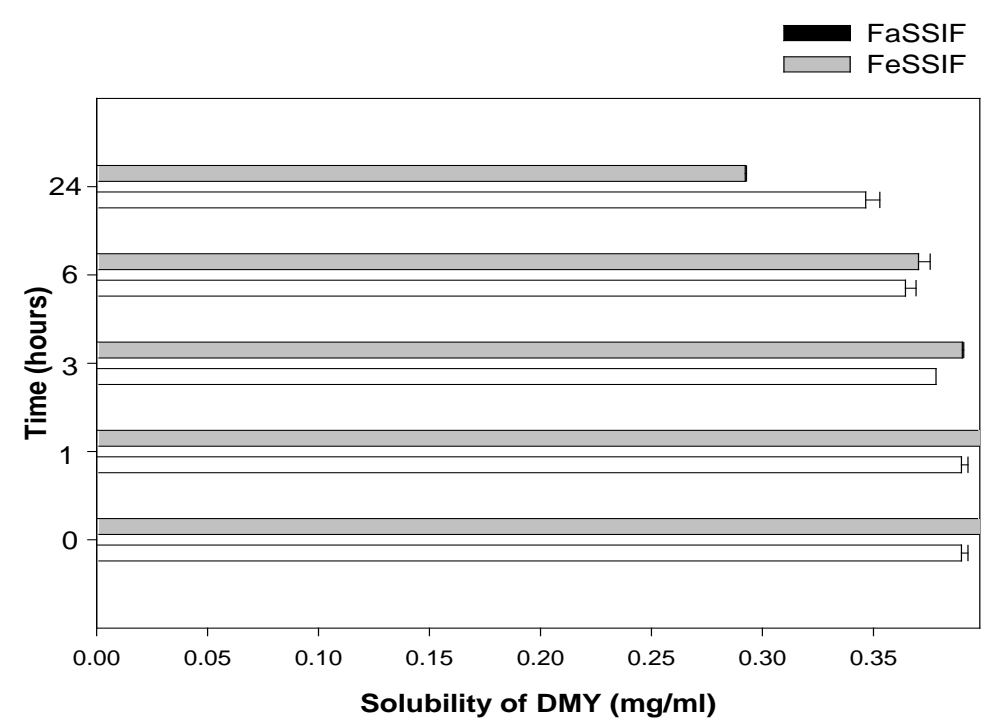

Fig. 14: Effect of bile salt-lecithin mixed micelles on the solubilization capacity of Miglyol 812/Imwitor 988-Tagat T0 \{70(70/30)30\} system after dispersion. 1g of each formulation containing approximately $40 \mathrm{mg}$ DMY was allowed to emulsify in $100 \mathrm{ml}$ FaSSIF (without bile salt mixed micelles) or FeSSIF medium which contained 15 mmol NaTC/3.75 mmol lecithin mixed micelles. Bars represent standard errors $(n=3)$

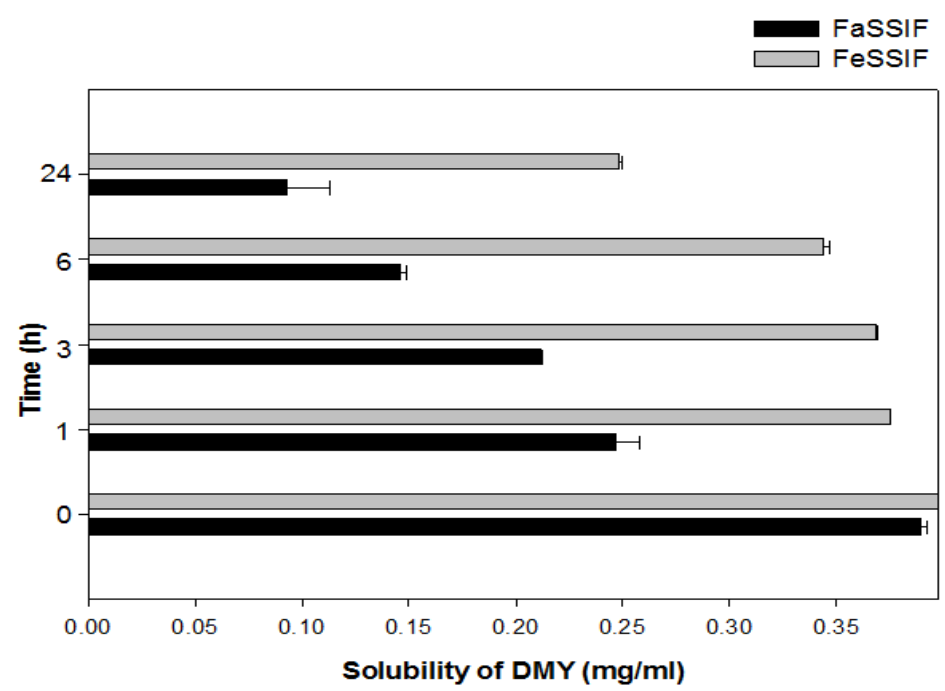

Fig. 15: Effect of bile salt-lecithin mixed micelles on the solubilization capacity of miglyol 812/Imwitor 988-Tagat T0 \{70(60/40)30\} system after dispersion in various emulsification media. Bars represent standard errors $(n=3)$ 


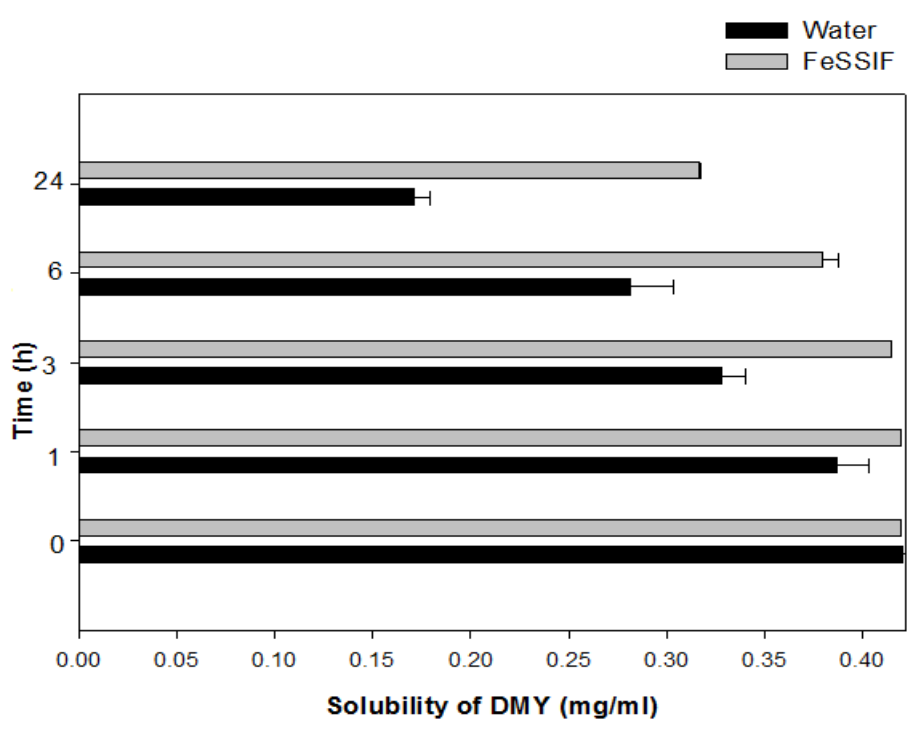

Fig. 16: Effect of bile salt-lecithin mixed micelles on the solvency of class IIIA lipid system containing a water-soluble cosolvent (Miglyol 812/Imwitor 988-Cremophor RH40-Ethanol 50(50/50)30/20) after dispersion in water or FeSSIF. Bars represent standard errors (n $=3$ )

Data obtained by Solomon et al. [40] for a range of steroids confirmed that shape is a factor which clearly can influence the extent of solubilization as the spatial orientation into the micelles available to the drug is limited. Long-chain esters of hydrocortisone such as hydrocortisone caprylate whilst being hydrophobic with log P 7.82 could not be incorporated into the micelles as efficiently as progesterone which is less hydrophobic (log P 4.22) and therefore, solubilization enhancement was found to be far more less in the former. Furthermore, the higher affinity of bile salt micelles for indomethacin as compared to phenylbutazone could not be explained on the basis of lipid solubility and molal volume. The lower interaction of phenylbutazone with bile salts was attributed to repulsion forces due to its carbon acid [41].

Fig. 14-16 depict the effect of various emulsification media on the solubilization behaviour of DMY from Type II and III lipid-class systems. For this study, FaSSIF medium was used without bile saltlecithin mixture while FeSSIF contained mixed micelles of $15 \mathrm{mmol}$ sodium taurocholate (NaTC)/3.75 mmol lecithin. For Miglyol 812/Imwitor 988 (7:3)-Tagat TO system, there was no significant difference in the solubilization profiles of DMY in FaSSIF vis-à-vis FeSSIF media for the first $6 \mathrm{~h}$ from the emulsification event, see fig. 14. This is because loss of solvent capacity of the resultant dispersion in FaSSIF medium started to occur significantly $24 \mathrm{~h}$ aftermath emulsification. Therefore, the effect of NaTC/lecithin mixed micelles in the solubilization enhancement of DMY could not be discerned. Nevertheless, the solubilization behaviour of DMY from Miglyol 812/Imwitor 988 (6:4)-Tagat T0 system was substantially influenced by emulsification in FaSSIF media, fig. 15. As was previously elaborated, gradual phase separation was observed after the dispersion of the former system in FaSSIF. Formation of an oil layer that is rich with hydrophobic surfactant and increasing amounts of dissolved DMY continued to develop on the top of the aqueous phase which contained few dispersed oil droplets. As a result, there had been drop in DMY solubility progressively with time after emulsification in FaSSIF. After $24 \mathrm{~h}$ from the emulsification event and due to oil separation, almost $75 \%$ drop of the initial DMY solubility was observed. However, when the emulsification medium contained bile salt-lecithin mixed micelles to stimulate the intestine at fed state as in FeSSIF, the resulting dispersion was able to keep DMY in solution almost up to $24 \mathrm{~h}$ after dispersion, see fig. 15. This increase in DMY solubility is attributed to the enhancement of oil solubilization within the bile salt micellar system. Similarly, as depicted in fig. 16, in contrast to the loss of solvent capacity of Miglyol 812/Imwitor 988-Cremophor RH40 system containing 20\% $\mathrm{w} / \mathrm{w}$ Ethanol after dispersion in water, the emulsification in FeSSIF maintained the solubility of drug in solution. This indicates the capacity of NaTC/lecithin mixed micelles to prevent the drug from precipitation in the lumen of the gut during digestion of lipid formulation. Despite the claim that the absorption of Cyclosporin A 'Sandimmune Neoral' is less affected by bile flow and pancreatin [42], it is anticipated that the role of mixed micelles must become evident in the pharmacokinetics of Neoral whereby, at least a single oral dose of $200 \mathrm{mg}$ might be needed. Therefore, there is an immense need for practical methods to predict the fate of drugs after the dispersion of lipid systems in the GI tract.

It is worth noting here that loss of solvent capacity for all dispersions in FeSSIF was only observed $24 \mathrm{~h}$ aftermath emulsification which might reflect the in-stability of mixed micelles with time. Yet, it is evident that the efficiency of dissolution and absorption for lipophilic compounds with $\log \mathrm{P}>2$ are probably better in the fed state than fasted. In a study by Charman et al. [43] in healthy human volunteers showed that both peak concentration and area under the curve were about three times higher when danazol was administered in fed than fasted conditions.

\section{CONCLUSION}

The bioavailability enhancement of most oral lipid-based formulations depends on the ability of the oil vehicle to maintain the drug in solution after dispersion. Solvency of emulsions formed by selfemulsifying drug delivery system is an important parameter influencing the fate of dissolved drug after dispersion of the formulation. Physicochemical factors which determine the solvent capacity of these emulsions such as, hydrophilicity of lipid system, droplet size of the resultant dispersion, $\log \mathrm{P}$ of drug, ionic strength of the emulsification media and the bile salt-lecithin mixed micelles were sought for investigating the tendency of drug to precipitate after administration of an oily vehicle. In vitro methods, however, were used in this study in order to investigate the solubilization behaviour of the drug from dispersions formed by Type II and Type III lipid class systems in an attempt to predict the dynamic changes, which are expected to occur in the gut. Nonetheless, there is a clear need for developing methods for tracking the solubilization state of the drug in vivo.

\section{ACKNOWLEDGMENT}

I am grateful to my supervisors Dr. Stephen Moss and Prof. Colin Pouton for their endless support, guidance and encouragement throughout this project. I am also grateful to the Department of Pharmacy and Pharmacology at Bath University for providing good atmosphere and excellent facilities of research. I am also thankful to Capsugel for their sponsorship and to Goldschmidt (Germany), BASF Corporation, Condea Chemie (Hüls), Gattefossé and Croda who have been providing us with the excipients needed. Finally, my gratitude's go to the school of Pharmacy at Applied Science Private University for their support. 


\section{AUTHORS CONTRIBUTIONS}

All the author have contributed equally

\section{CONFLICTS OF INTERESTS}

Author has none to declare

\section{REFERENCES}

1. Shah NH, Carvajal MT, Patel CI, Infeld MH, Malick AW. Selfemulsifying drug delivery system (SEDDS) with polyglycolyzed glycerides for improving in vitro dissolution and oral absorption of lipophilic drugs. Int J Pharm 1994;106:15-23.

2. Farah N, De Teddeo M, Larfret JP, Denis J. Self-microemulsifying drug delivery system for improving in vitro dissolution of drugs. AAPS Annual Meeting, Orlando, FL; 1993.

3. Pouton CW. Lipid formulations for oral administration of drugs: non-emulsifying, self-emulsifying and "selfmicroemulsifying" drug delivery systems. Eur J Pharm Sci 2000;11:93-8.

4. Pouton CW. Formulation of poorly water-soluble drugs for oral administration: physicochemical and physiological issues and the lipid formulation classification system. Eur J Pharm Sci 2006;29:278-87.

5. Pouton CW. Key issues when formulating hydrophobic drugs with lipids. Bulletin Technique Gattefosse; 1999.

6. Suman K, Chandrasekhar P, Balaji S. Approaches for the development of solid self-emulsifying drug delivery systems and dosage forms. Asian J Pharm Sci 2009;4:240-53.

7. Savla R, Browne J, Plassat V, Wasan KM, Wasan EK. Review and analysis of FDA approved drugs using lipid-based formulations. Drug Dev Ind Pharm 2017;43:1743-58.

8. Crew M. Bioavailability enhancement-analysis of the historical use of solubilization technologies. Drug Dev Delivery 2014. Available from: http://www.drugdev.com/Main/BackIssues/bioavailabilityenhancement. [Last accessed on 20 Jun 2018]

9. Strickley RG. Currently marketed oral lipid-based dosage forms: drug products and excipients. In: Hauss D. editor. Oral lipid based formulations. 1st ed. New York: Informa Healthcare; 2007.

10. Wishart DS, Knox C, Guo AC, Shrivastava S, Hassanali M, Stothard P, et al. DrugBank: a comprehensive resource for in silico drug discovery and exploration. Nucleic Acids Res 2006;1:D668-72.

11. Strickley RG. Solubilizing excipients in oral and injectable formulations. Pharm Res 2004;21:201-30.

12. Agrawal SGT, Tripathi DK, Alexander A. A review on novel therapeutic strategies for the enhancement of solubility for hydrophobic drugs through lipid and surfactant based self micro emulsifying drug delivery system: a novel approach. Am J Drug Discovery Dev 2012;2:143-83.

13. Webster GF, Leyden JJ, Gross JA. Comparative pharmacokinetic profiles of a novel isotretinoin formulation (isotretinoin-lidose) and the innovator isotretinoin formulation: a randomized, 4treatment, crossover study. J Am Acad Derma 2013;69:762-7.

14. Bennett LL, Ingason A. Enzalutamide (Xtandi) for patients with metastatic, resistant prostate cancer. Annals Pharmacol 2014;48:530-7.

15. Fukihara J, Kondoh Y. Nintedanib (OFEV) in the treatment of idiopathic pulmonary fibrosis. J Exp Rev Respir Med 2016;10;1247-54.

16. Sprague SM, Strugnell SA, Bishop CW. Extended-release calcifediol for secondary hyperparathyroidism in stage 3-4 chronic kidney disease. Expert Rev Endocrinol Metab 2017;12:289-301.

17. Hasan NM. Role of medium-chain fatty acids in the emulsification mechanistics of self-micro-emulsifying lipid formulations. Saudi Pharm 2014;22:580-90.

18. Hasan MYN. Self-micro-emulsifying lipid formulations to improve the bioavailability of poorly water-soluble drugs. $\mathrm{Ph}$. D. thesis, University of Bath; 2004.

19. Galia E, Nicolaides E, Horter D, Lobenberg R, Reppas C, Dressman JB. Evaluation of various dissolution media for predicting in vivo performance of class I and class II drugs. Pharm Res 1998;15:698-705.
20. Yalkowsky SH, Flynn G, Amidon G. Solubility of non-electrolytes in polar solvents. J Pharm Sci 1972;61:983-4.

21. Yalkowsky SH, Valvani S, Amidon G. Solubility of nonelectrolytes in polar solvents IV: non-polar drugs in mixed solvents. J Pharm Sci 1976;65:1488-93.

22. Solubilization of drugs by cosolvents, Techniques of solubilization of drugs: Edited by Yalkowsky SH, Marcel Dekker. New York; 1981. p. 91-134.

23. Hasan NMY. Effect of a model lipophilic compound on the phase behaviour of hydrophilic self-micro-emulsifying lipid formulations. J Pharm Res 2016;10:647-54.

24. Van HP, Liu X, Fahr A. Drug delivery strategies for poorly water-soluble drugs: the industrial perspective. Expert Opin Drug Delivery 2011;8:1481-500.

25. Hasan NMY, Hayajneh FM, Khaleel MA, Alharthi SA, Shahada HM, Almalki HF. Development of potential selfmicroemulsifying lipid formulation for the oral administration of curcumin. Int J Adv Pharm Biol Chem 2015;4:590-602.

26. Sawicki E, Schellens JHM, Beijnen JH, Nuijen B. Inventory of oral anticancer agents: pharmaceutical formulation aspects with focus on the solid dispersion technique. Cancer Treat Rev 2016;50:247-63.

27. Myers RA, Stella VJ. Factors affecting the lymphatic transport of penclomedine (NSC-338720), a lipophilic cytotoxic drug: comparison to DDT and hexachlorobenzene. Int J Pharm 1992;80:51-62.

28. Charman WN, Stella VJ. Estimating the maximal potential for intestinal lymphatic transport of lipophilic drug molecules. Int J Pharm 1986;34:175-8.

29. Porter $\mathrm{CJH}$, Charman WN. In vitro assessment of oral lipid based formulations. Adv Drug Delivery Rev 2001;50:127-47.

30. Dressman JB, Reppas C. In vitro-in vivo correlations for lipophilic, poorly water-soluble drugs. Eur J Pharm Sci 2000;11:73-80.

31. Malik NA. Solubilization and interaction studies of bile salts with surfactants and drugs: a review. Appl Biochem Biotechnol 2016;179:179-201.

32. Naylor LJ, Bakatselou V, Dressman JD. Comparison of the mechanism of dissolution of hydrocortisone in simple and mixed micelle systems. Pharm Res 1993:10:865-70.

33. Hoogevest PV. Review-an update on the use of oral phospholipid excipients. Eur J Pharm Sci 2017;108:1-12.

34. Khadra I, Zhou Z, Dunn C, Wilson CG, Halbert G. Statistical investigation of simulated intestinal fluid composition on the equilibrium solubility of biopharmaceutics classification system class II drugs. Eur J Pharm Sci 2015;67:65-75.

35. Zhou Z, Dunn C, Khadra I, Wilson CG, Halbert GW. Statistical investigation of simulated fed intestinal media composition on the equilibrium solubility of oral drugs. Eur J Pharm Sci 2017;99:95-104.

36. Shankland $W$. The equilibrium and structure of lecithin-cholate mixed micelles. Chem Phys Lipids 1970;4:109-30.

37. Magee GA, French J, Gibbon B, Luscombe C. Bile salt/lecithin mixed micelles optimized for the solubilization of a poorly soluble steroid molecule using statistical experimental design. Drug Dev Indus Pharm 2003;29:441-50.

38. Amidon GL, Choe SY, Vieira M, Oh DM. Solubility, intrinsic dissolution and solubilization: influence on absorption. In: Amidon GL, Robinson JR, Williams RL. Eds. Scientific foundations for regulating drug product quality, AAPS Press, Alexandria; 1997. p. 99-113.

39. Mithani SD, Bakatselou V, Tenhoor CN, Dressman JD. Estimation of the increase in solubility as a function of bile salt concentration. Pharm Res 1996;13:163-7.

40. Solomon LJ, Embleton JK, Pouton CW. Solubilization of steroidal compounds by mixed bile salt/lecithin micelles. Eur Symposium: Formulation Poorly-Available Drugs For Oral Administration 1996;2:219-22.

41. Miyazaki S, Yamahira T, Morimota Y, Nadia T. Micellar interaction of indomethacin and phenylbutazone with bile salts. Int J Pharm 1981;8:303-10. 
42. Trull $A K$, Tan KKC, Uttridge I, Bauer $R$, Alexander $G$, Jamieson NV. Cyclosporine absorption from microemulsion formulation in liver transplant recipient. Lancet 1993;341:433.
43. Charman WN, Rogge MC, Boddy AW, Barr WH, Berger BM Absorption of danazol after administration to different sites of the gastrointestinal tract and the relationship to single and double peak phenomena in the plasma profiles. J Clin Pharmacol 1993;33:1207-13. 